\section{Should Influenza Immunization Be Mandatory for All Health Care Providers?}

\section{THE "PRO" SIDE}

"First, do no harm." This famous line is from the Hippocratic Oath, which all physicians swear to uphold when they enter their profession. Other health care professionals commit to similar oaths, including pharmacists, who begin their careers by swearing to the Oath of Maimonides. All of these statements commit those of us working in these various health care professions to putting our patients first when they interface with us in care. These commitments have now expanded to an increased focus in institutional practice on patient- and family-centred care. If patients come first, then why is there not a universal commitment to immunization as a patientcentred strategy?

Is it because influenza is a disease that doesn't cause harm or that's easily treatable? Seasonal influenza affects millions of Canadians each year, resulting in hospital admission for more than 12000 people and causing death in 3500 patients. ${ }^{1}$ These are significant numbers, and they do not even begin to address the economic impact of the flu on those Canadians who miss school or work as a result of the milder forms of the infection. The most vulnerable in society, including elderly and immunocompromised patients, are most at risk of morbidity and mortality. Influenza is highly infectious and can be transmitted for up to 2 days before an individual even knows he or she is infected. This means that health care workers can spread the virus even if they feel completely well. The presence of stockpiles of oseltamivir have suggested to many health care workers that effective methods are available to treat these patients and even to provide prophylaxis to patients or staff who may have been in contact with the virus. The Cochrane Collaboration has worked hard for several years to find out if oseltamivir is really effective. The makers of the drug withheld original study data for a long time; however, these data were recently released, and in April 2014 the BMJ published a systematic review of the benefits of oseltamivir (also published in the Cochrane Library), which confirmed mild benefits with treatment (i.e., symptom reduction of less than a day), but no reductions in hospital admissions or transmission of the virus. ${ }^{2}$ With these outcomes came increased risks of nausea, vomiting, headaches, and psychiatric symptoms. ${ }^{2}$ Although the findings were criticized because the study considered only randomized controlled studies and not subsequent observational trials during the pandemic flu season, they speak to the limited utility of oseltamivir when used in the population of healthy health care workers.

Returning to the issue of why universal commitment to immunization is lacking, is it because the influenza vaccine is ineffective? If the infection is real, and there are no safe and effective treatment or prophylaxis strategies available, then perhaps the reason that health care workers refuse the vaccine is because it is not effective. Studies published from 1943 to 1969 suggested influenza vaccine effectiveness of $70 \%$ to $90 \%$. ${ }^{3}$ However, these numbers did not reflect true immunity, as they were based on different vaccine types, including whole-virus vaccines early on, and used hemaglutinin antibody titres as a surrogate marker for infection. It was later shown that, upon exposure to influenza virus, these antibody titres increased to a much lesser extent in those who had been vaccinated than in unvaccinated individuals. ${ }^{3}$ More recently, studies examining confirmation of infection by polymerase chain reaction have revealed that the effectiveness of influenza vaccine (i.e., trivalent inactivated vaccine) is about $62 \%$ in adults 18-64 years of age. ${ }^{4}$ Immunity is even less among the elderly. While all agree there is a need to develop more effective vaccines, it appears clear that immunizing health care workers may protect not only our own workforce, but also the vulnerable patients in our care. A protection level of $62 \%$ far outweighs the reduction in our own symptoms of less than 1 day that would be achieved with antiviral therapy and the potential morbidity and mortality our patients may experience from our lack of protection.

The last remaining question to be answered is why it would not be worth the potential of at least moderate protection from infection for ourselves and protection from harm for our patients, unless it is because the vaccine carries great risk to our personal health. The adverse effects associated with influenza vaccine are generally considered to be mild soreness at the site of injection, with no increase in fever or other systemic symptoms, relative to placebo. Rarely reported events include oculorespiratory syndrome, which was associated with the influenza vaccine of 2000/2001 in particular and is generally considered a mild, self-limited adverse effect. Egg allergy is no longer considered a contraindication for use of the trivalent inactivated vaccine, and individuals with such an allergy can be safely vaccinated. The adverse effect that appears to raise the greatest concern is Guillain-Barré syndrome. 
A common question among those who decline immunization is, "Why should I risk a serious side effect like Guillain-Barré syndrome for a vaccine that may only be $60 \%$ effective?" This question reveals the common perception that the adverse effects of influenza vaccine are worse than the disease itself. The complications of influenza include pneumonia, secondary bacterial pneumonia, pneumonia due to unusual pathogens, and exacerbations of chronic pulmonary diseases. In addition, myositis and rhabdomyolysis have been observed with influenza $\mathrm{A}$ and influenza B. Neurologic complications of influenza include encephalopathy, encephalomyelitis, transverse myelitis, aseptic meningitis, focal neurologic disorders, and Guillain-Barré syndrome itself (which represents the most common form of acute flaccid paralysis and is often associated with viral illness ${ }^{5,6}$ ). It can be difficult to assess the rates of complications of influenza illness relative to the rates for adverse events of the vaccine without careful estimation of background disease rates and the associated risk of Guillain-Barré syndrome with influenza. Several studies have been conducted using adverse event data collected during the 2009 pandemic, as the adjuvanted vaccine was newly released at that time and was closely scrutinized. Poland and others ${ }^{7}$ studied US data from the pandemic and found a significantly lower incidence of Guillain-Barré syndrome, hospital admissions, and death among those who had been immunized. From an epidemiologic standpoint, even if a vaccine is only $60 \%$ effective, a high immunization rate has the potential to remove a substantial number of people from the susceptible population. This can in turn limit the spread of the disease and protect a greater number of people from the mortality and morbidity associated with the disease itself.

So, if none of these reasons are holding health care workers back from immunization, then why are rates so low? No voluntary influenza immunization program has achieved rates much higher than $70 \%$, a rate too low to guarantee any level of herd immunity. ${ }^{8}$ Conversely, sites like Virginia Mason Medical Center in Seattle, Washington, have taken the approach that influenza immunization is a duty of care to patients and have mandated immunization for all of their employees, physicians, contractors, and volunteers. Under this program, more than 10 years old at this point, they have managed annual immunization rates approaching $100 \% .{ }^{9}$

Studies such as these provide the basis for the Society for Healthcare Epidemiology of America (SHEA) to endorse "a policy in which annual influenza vaccination is a condition of both initial and continued health care professional employment and/or professional privilege"..$^{10}$ It is also the basis for the newly released statement on influenza immunization for health care workers of the Canadian Society of Hospital Pharmacists. ${ }^{11}$ Unvaccinated health care professionals place themselves and their patients at risk for influenza. For this reason, the SHEA guidelines recommend masks for all hospital workers who have not been immunized. British Columbia and Saskatchewan recently implemented mandatory influenza immunization for their health care workers. Without greater support for voluntary immunization, we can expect to see such mandatory programs spread across the country. Get your flu shot, not the flu.

\section{References}

1. Public health reminder: seasonal flu. Ottawa (ON): Public Health Agency of Canada; updated 2015 Jan 5 [cited 2015 Jan 6]. Available from: www.phac-aspc.gc.ca/phn-asp/2014/flu-grippe-1022-eng.php

2. Jefferson T, Jones M, Doshi P, Spencer EA, Onakpoya I, Heneghan CJ. Oseltamivir for influenza in adults and children: systematic review of clinical study reports and summary of regulatory comments. BMJ. 2014; 348:g2545.

3. Ohmit SE, Petrie JG, Cross RT, Johnson E, Monto AS. Influenza hemagglutination-inhibition antibody titer as a correlate of vaccine-induced protection. J Infect Dis. 2011;204(12):1879-85.

4. Osterholm MT, Kelley NS, Sommer A, Belongia EA. Efficacy and effectiveness of influenza vaccines: a systematic review and meta-analysis. Lancet Infect Dis. 2012;12(1):36-44. Erratum in: Lancet Infect Dis. 2012;12(9):655.

5. Studahl M. Influenza virus and CNS manifestations. J Clin Virol. 2003;28(3):225-32.

6. Foulkes W, Rees J, Sewry C. Influenza A and rhabdomyolysis. I Infect. 1990;21(3):3034.

7. Poland GA, Poland CM, Howe CL. Influenza vaccine and Guillain-Barré syndrome: making informed decisions. Lancet. 2013;381(9876):1437-9.

8. Lam PP, Chambers LW, MacDougall DM, McCarthy AE. Seasonal influenza vaccination campaigns for health care personnel: systematic review. CMAJ. 2010;182(12):E5428.

9. Rakita RM, Hagar BA, Crome P, Lammert JK. Mandatory influenza vaccination of healthcare workers: a 5-year study. Infect Control Hosp Epidemiol. 2010;31(9):881-8.

10. Talbot TR, Babcock H, Caplan AL, Cotton D, Maragakis LL, Poland GA, et al. Revised SHEA position paper: influenza vaccination of healthcare personnel. Infect Control Hosp Epidemiol. 2010;31(10):987-95.

11. Influenza immunization for healthcare workers: statement. Ottawa $(\mathrm{ON})$ : Canadian Society of Hospital Pharmacists; 2014 [cited 2015 Jan 6]. Available from: www.cshp.ca/productsServices/officialPublications/type_e.asp\# statements

\section{Margaret Gray, BSP, FCSHP \\ Clinical Practice Manager (North) \\ Pat Mayo, BScPharm, PhD \\ Clinical Practice Leader (North) \\ Alberta Health Services \\ Edmonton, Alberta}

Competing interests: Margaret Gray is a contributor to, and supporter of, the position statement of the Canadian Society of Hospita Pharmacists on influenza immunization for health care workers (cited as reference 11 in this article). No other competing interests declared.

\section{THE "CON" SIDE}

There is much ongoing debate about the need for mandatory approaches to increase rates of immunization against seasonal influenza among health care providers. Although there is general agreement and supporting evidence that mandatory programs do 
indeed increase immunization rates, many health care providers question whether these high rates actually achieve the lofty outcomes sought by such policies. Does immunization against the influenza virus actually decrease rates of viral transmission from health care providers to patients and vice versa? Does the evidence justify the humanistic impact of heavy-handed, top-down, Big Brother-like edicts? Or could high rates of immunization be achieved through other, more palatable approaches?

As a pharmacy director tasked with ensuring that all health care providers in my realm of influence are immunized against influenza every flu season, I frequently hear from staff members articulating their concerns as to why mandatory influenza immunization programs may not be in the best interests of patients or staff. These concerns include a lack of direct evidence for effectiveness of the vaccine; an unjustified infringement on religious customs, personal rights, or privacy of medical information; a belief in the existence of a government and/or pharmaceutical industry conspiracy; and a fear of serious personal harm.

The most difficult science-based challenge articulated by health care providers who oppose influenza immunization, especially in the acute care sector, is the lack of direct evidence showing that mass immunization of health care providers will indeed reduce the rate of transmission of the influenza virus to the patients that they care for. The protection conferred by immunization against influenza infection is argued to be at best an inexact science. The composition of the upcoming season's influenza vaccine must be determined annually on the basis of predictions as to which viral strains are likely to spread, and these "guesstimates" may provide effectiveness rates of only $60 \%{ }^{1}$ Furthermore, it is argued that even if immunization can decrease the risk of progression to an influenza-like illness following exposure to the influenza virus, there are no data showing that this reduction in risk will then translate to the same degree of risk reduction for transmission of the virus from the health care provider to the patient. Current justification for proceeding with mass immunization of health care providers is based largely on mathematical modelling, rather than on hard evidence. ${ }^{2}$ In 2010, the Cochrane Collaboration published an analysis of about 50 controlled studies in which influenza vaccine was administered to healthy adults. ${ }^{3}$ The authors concluded that where the vaccine matched the circulating viral strain, the absolute difference in risk of subsequently developing influenza-like illness was only 3\% ( $4 \%$ in unvaccinated participants versus $1 \%$ in vaccinated participants); it was of course lower in situations where the circulating viral strain did not match the vaccine. The authors also concluded that there was a lack of evidence to support the contention that influenza immunization actually reduces the risk of viral transmission from individual to individual (which is the primary basis justifying mass immunization of health care providers). Then again, this "lack of evidence" may simply reflect the logistic and ethical challenges associated with designing and implementing such a study.
The most challenging nonscientific reasons encountered are infringements on an individual's right to personal choice, religious freedoms, or privacy of medical information. How can an industry-wide policy, based largely on the principle of "likelihoods" instead of evidence, be allowed to compromise fundamental human rights? Health care providers correctly argue that they are members of society in good standing who embrace their right to make personal health care choices. Many also hold religious beliefs that prohibit unnatural interventions such as vaccination. Furthermore, implementation of mandatory programs could entail the sharing of health care providers' personal health information (i.e., their influenza immunization history) beyond traditional medical spheres, to their direct supervisors and potentially other administrators. Under what dire criteria is this justified, they ask? Shouldn't less invasive interventions, such as hand-washing, be considered ahead of mandatory immunization? Does a conspiracy or "secret deal" exist between governments and the pharmaceutical industry to experiment with the public or to share profits? Will the existence of mandatory immunization programs undermine the incentive for the pharmaceutical industry to aggressively develop more effective vaccines and better immunization technology?

Interventions of any kind need to balance benefits against any known detriments. Do the claimed benefits of a mandatory influenza immunization program (decreased sickness rate among health care providers and decreased rate of transmission of influenza to patients) outweigh the potential detriments (inconvenience for health care providers, pain at the injection site, needlephobia, and potential for systemic side effects or adverse drug events)? Aside from needle-phobia and local reactions at the injection site-some of which can be quite uncomfortable and prolonged - many health care providers fear serious allergic reactions or the triggering of systemic or neurologic adverse events, such as Guillain-Barré syndrome. Although an association of the influenza vaccine with Guillain-Barré syndrome has now been largely dismissed, the fear of serious adverse consequences related to the vaccine remains. ${ }^{4}$

Health care providers argue that they possess the expert knowledge required to make the right choices for their patients and for themselves. Can they not be trusted to recognize potentially compromising situations and to act in the best interests of their patients? Why would they not endeavour to maintain their own good health in order to avoid exposing their patients to potential harm?

Personally, I am a believer in the benefits of the influenza vaccine and support efforts that encourage $100 \%$ immunization rates. However, I am concerned about the backlash associated with mandatory approaches and the potential risk of regression rather than progression. Mandatory approaches serve to unintentionally undermine the trust that we have in our staff, to put into question their core values and ethics, and to remove the opportunity for 
health care providers to choose to do the right thing. Understandably, they ask, "Could there not be another way?"

\section{References}

1. Osterholm MT, Kelley NS, Sommer A, Belongia EA. Efficacy and effectiveness of influenza vaccines: a systematic review and meta-analysis. Lancet Infect Dis. 2012;12(1):36-44. Erratum in: Lancet Infect Dis. 2012;12(9):655.

2. van den Dool C, Bonten MJ, Hak E, Wallinga J. Modeling the effects of influenza vaccination of health care workers in hospital departments. Vaccine. 2009;27(44):6261-7.

3. Jefferson T, Di Pietrantonj C, Rivetti A, Bawazeer GA, Al-Ansary LA, Ferroni E. Vaccines for preventing influenza in healthy adults. Cochrane Database Syst Rev. 2010;(7):CD001269.
4. Poland GA, Poland CM, Howe CL. Influenza vaccine and Guillain-Barré syndrome: making informed decisions. Lancet. 2013;381(9876):1437-9.

Mits Miyata, BSc(Pharm), ACPR, FCSHP

Pharmacy Director

Lower Mainland Pharmacy Services

Port Moody, British Columbia

Competing interests: Mits Miyata is a contributor to, and strong supporter of, the 2014 position statement of the Canadian Society of Hospital Pharmacists on influenza immunization for health care workers. No other competing interests declared.

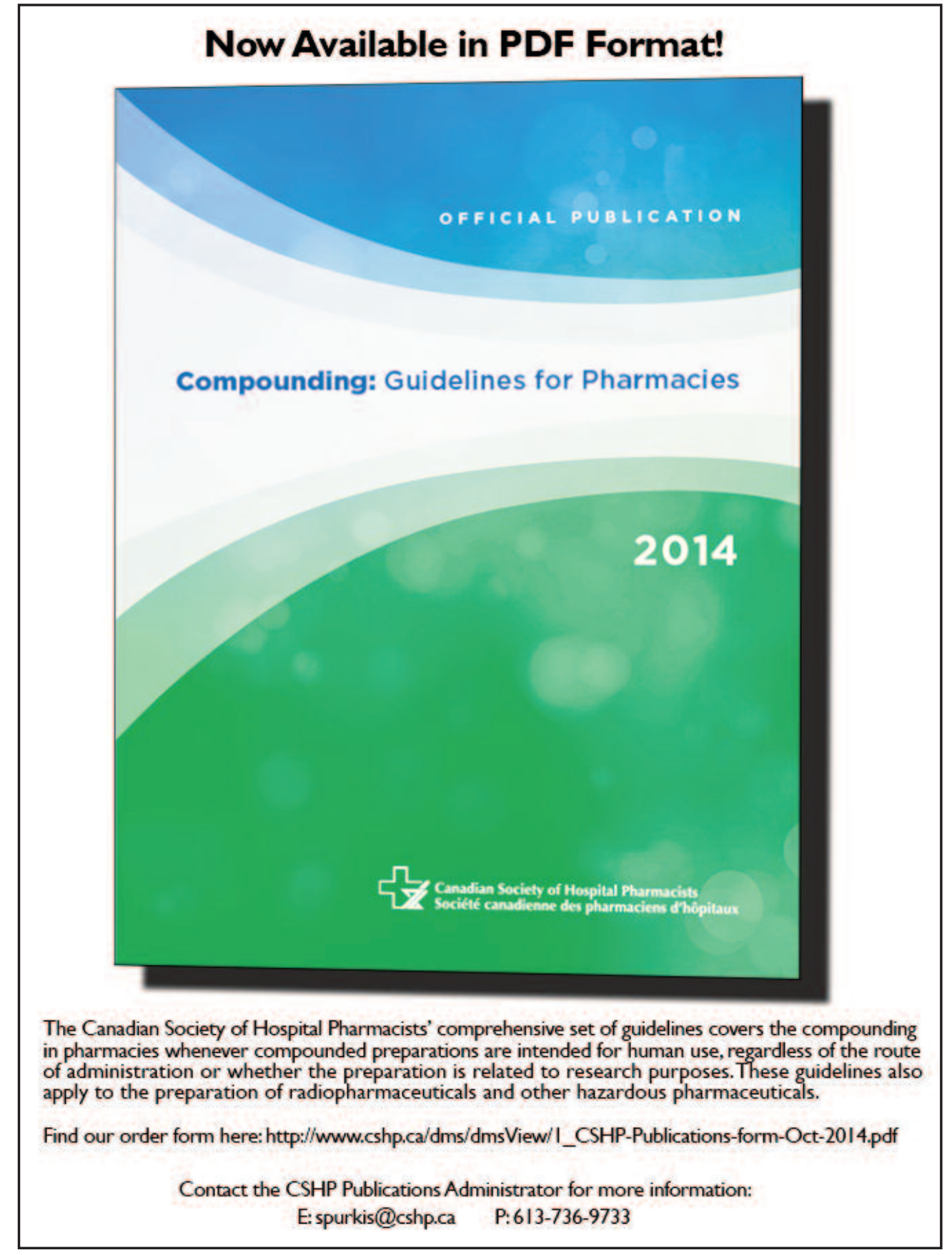

